Pacific Journal of Mathematics

CANCELLING 1-HANDLES AND SOME TOPOLOGICAL 


\title{
CANCELLING 1-HANDLES AND SOME TOPOLOGICAL IMBEDDINGS
}

\author{
Michael H. Freedman
}

\begin{abstract}
In this note we use the existence of a certain type of handle decomposition (see corollary) for compact simply connected P. L. 4-manifolds and R. Edwards results on the double suspension conjecture to prove:
\end{abstract}

THEOREM 2. Let $\alpha \in H_{2}(M ; Z)$ where $M$ is a compact simply connected P. L. 4-manifold. Then there is a proper topological imbedding (possible nonlocally flat) $\theta: S^{2} \times R \rightarrow M \times R$ (mapping ends to ends) with $\theta_{*}\left[S^{2} \times R\right]=\bar{\alpha} \in H_{2}(M \times R ; Z) . \quad \bar{\alpha}$ is the image of $\alpha$ under $\times R$. Proper, here, means inverse images of compact sets are compact.

In [2], we considered the problem of constructing smooth proper imbeddings, $\theta$, and showed that if $\alpha$ is characteristic (dual to $w_{2}(\tau(M))$, the only obstruction to the existence of $\theta$ is an Arf invariant which is equal to the Milnor-Kervaire number (=(signature $(M)-\alpha \cdot \alpha / 8)(\bmod 2))$ when $M$ is closed and that if $\alpha$ is ordinary (not dual to $W_{2}(\tau(M))$ there is no obstruction. This suggests two problems: (1) Can $\theta$ always be arranged to be topologically locally flat, and (2) can $\theta$ always be arranged to be P. L.?

Here is our "handle cancellation" theorem:

THEOREM 1. Let $M$ be any compact connected P. L. manifold of dimension $=m$ (assume $M$ orientable if $m=3$ ). Let $N$ be a compact connected codimension 0 submanifold of $\partial M$. If $\pi_{1}(M, N)=0$, then there is a codimension 0 submanifold, $\bar{N}$, of $M$ with: (1) $N \hookrightarrow \bar{N}$, (2) the inclusion $N \hookrightarrow \bar{N}$ is a homotopy equivalence, (3) $M=\bar{N} \cup$ 2-handles $\cup 3$-handles $\cup \cdots \cup m$-handles.

Note. The P. L. category is convenient here since handle decompositions always exists.

Proof. If $n \geqq 5$, the usual arguments for cancelling handles produce the desired $\bar{N} \stackrel{\text { P. L. }}{=} N \times I$ (see Appendix [3]). We need only consider the cases $m=3$ or 4 .

Let $m=4$ and let $\mathscr{H}(M, N)$ be a handle decomposition of $M$ relative to $N$. We may assume $\mathscr{H}(M, N)$ has no zero-handles.

Let $\left\{h_{i}^{1}\right\}=\left\{D_{i}^{1} \times D_{i}^{m-1}\right\}$ be the 1-handles. Let $\left\{c_{i}\right\}$ be closed curves 
on $L_{1}$, the level after the 1-handles are attached, each consisting of $\left(D_{i}^{1} \times\right.$ pt.) for some pt. $\in \partial D_{i}^{m-1}$ and an arc in $N-\left\{D_{i}^{1} \times \partial D_{i}^{m-1}\right\}$. We claim that the latter arcs may be chosen so that each curve, $c_{i}$, is null homotopic in $X \stackrel{\text { def }}{=} \overline{M-(1-\text { handles of } \mathscr{H}(M, N))}$. Since $m=4$, $\pi_{1}(X) \rightarrow \pi_{1}(M)$ is an isomorphism. The arcs may be chosen (since $\pi_{1}(N) \rightarrow \pi_{1}(M)$ is epic) so that each $c_{i}$ represents $0 \in \pi_{1}(M)$ and, therefore, $0 \in \pi_{1}(X)$.

Let $\left\{\gamma_{j}\right\}$ be the disjoint simple closed curves in $L_{1}$ along which the 2-handles $\left\{h_{j}\right\}$ are attached. Picking paths to the base pt., *, $\left\{\gamma_{i}\right\}$ determines relations $\left\{r_{j}\right\}$ and $\pi_{1}(X)=\pi_{1}\left(L_{1}\right) /\left\langle r_{j}\right\rangle$. Choosing a path from $c_{1}$ to $*$, we have $\left[c_{i}\right] \in\left\langle r_{j}\right\rangle$. So $\left[c_{i}\right]=\prod_{k=1}^{n} u_{k} x_{k} u_{k}^{-1}$ where $u_{k} \in \pi_{1}(L)$ and $x_{k} \in\left\{r_{j}, r_{j}^{-1}\right\}$. For each curve $c_{i}$, introduce a trivial oriented (2-handle, 3-handle) pair. Let $h_{i}^{2}$ be the new 2-handle. Choose a path from $\partial h_{i}^{2}$ to $*$. Now perform a sequence of $n$-handle passings. $h_{i}^{2}$ should be passed over the oriented ( + or - as $x_{k}=r_{j}$ or $r_{j}^{-1}$ ) 2-handles corresponding to $x_{1}, \cdots, x_{n}$ along arcs corresponding to the elements $u_{1} \cdots u_{n}$. The framing along each arc is immaterial so long as it restricts at the end points to a framing induced by the orientation of each 2-handle. Let $\left\{\gamma_{i}\right\}$ be the curves along which $\left\{h_{i}^{2}\right\}$ are attached after the above handle passings. $\gamma_{i}$ is homotopic to $c_{i}$. By the handle cancellation lemma [3], attaching 2-handles to $\left\{c_{i}\right\}$ would result in a product $N \times I$. Since homotopy type depends only on the homotopy class of attaching maps, $\bar{N} \stackrel{\text { def }}{=} N \cup\left\{h_{i}^{1}\right\} \cup$ $\left\{h_{i}^{2}\right\} \stackrel{\text { h.e. }}{\simeq} N \times I . \quad \bar{N}$ has the desired properties.

Let $m=3$. If $\pi_{1}(N)=0$ then $\pi_{1}(M)=0$ and $M$ must be a homotopy ( $S^{3}$-interior of closed disks). Let $\bar{N}=\overline{M-\text { closed disk } U}$ thickened ares to $\partial$ components $\not \supset N\}$, so $M=\bar{N} \cup 2$-handles $\cup 3$ handles. We now assume $\pi_{1}(N) \neq 0$.

If $\pi_{1}(N) \rightarrow \pi_{1}(M)$ is an isomorphism, every imbedded 2-sphere in $M$ seperates $M$, one component of the complement being a homotopy $B^{3}$ with finitely many punctures. Let $\bar{M}=M$

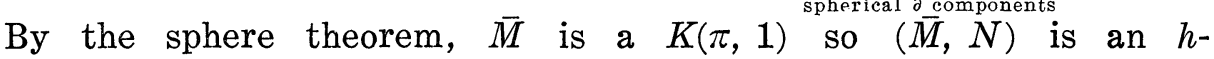
cobordism. But $M \stackrel{\text { diff }}{=} \bar{M} \cup 2$-handles, so $\bar{M}$ satisfies the conditions for $\bar{N}$.

Assume $\pi_{1}(N) \rightarrow \pi_{1}(M)$ is epi. By Dehn's lemma, if $\pi_{1}(N) \rightarrow \pi_{1}(M)$ is not injective, there is an essential simple closed curve, $\alpha \subset N$, bounding an imbedded 2-disk $\beta \subset M$. Let $\left(M^{\prime}, N^{\prime}\right)$ be the result of ambient surgery (handle subtraction) along $\beta . \quad \pi_{0}\left(N^{\prime}\right) \rightarrow \pi_{0}\left(M^{\prime}\right)$ is an isomorphism. (Proof: $\beta(\alpha)$ disconnects $M(N)$ if and only if there is no curve in $M(N)$ meeting $\beta(\alpha)$ algebraically once. Since $H_{1}(N) \rightarrow$ $H_{1}(M)$ is epi, there is a dual curve for $\beta$ if and only if there is a dual curve for $\alpha$.) 
Proposition. On each component, $\pi_{1}\left(N^{\prime}\right) \rightarrow \pi_{1}\left(M^{\prime}\right)$ is epi.

Proof. $M$ is obtained from $M^{\prime}$ by attaching a 1-handle, and $N^{\prime}$ is obtained from $N$ by the corresponding 0 -surgery. The proposition can be deduced from the following group theoretic fact: Let $\theta: A \rightarrow X, \phi: B \rightarrow Y$ be group homomorphisms. If $\theta * \phi$ is epi, then $\theta$ and $\phi$ are epi.

Proceeding inductively (on the genus of the components of $N^{\prime}$ ), we obtain $\left(M^{\prime \prime}, N^{\prime \prime}\right)$ with $\pi_{1}\left(N^{\prime \prime}\right) \rightarrow \pi_{1}\left(M^{\prime \prime}\right)$ an isomorphism on each component. This decomposes $M$ as:

\section{Diagram 1:}

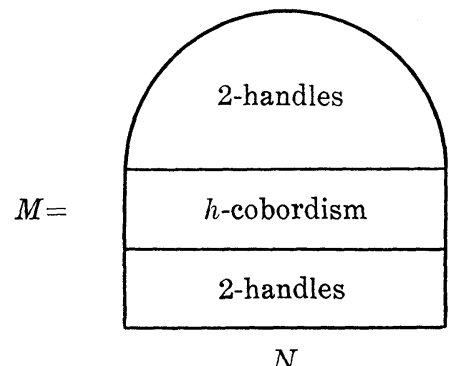

By a theorem of J. Stallings [5], every $h$-cobordism between orientable surfaces is the connected sum of a product and a homotopy 3 -sphere, $\Sigma^{3}$. So we have:

\section{Diagram 2:}

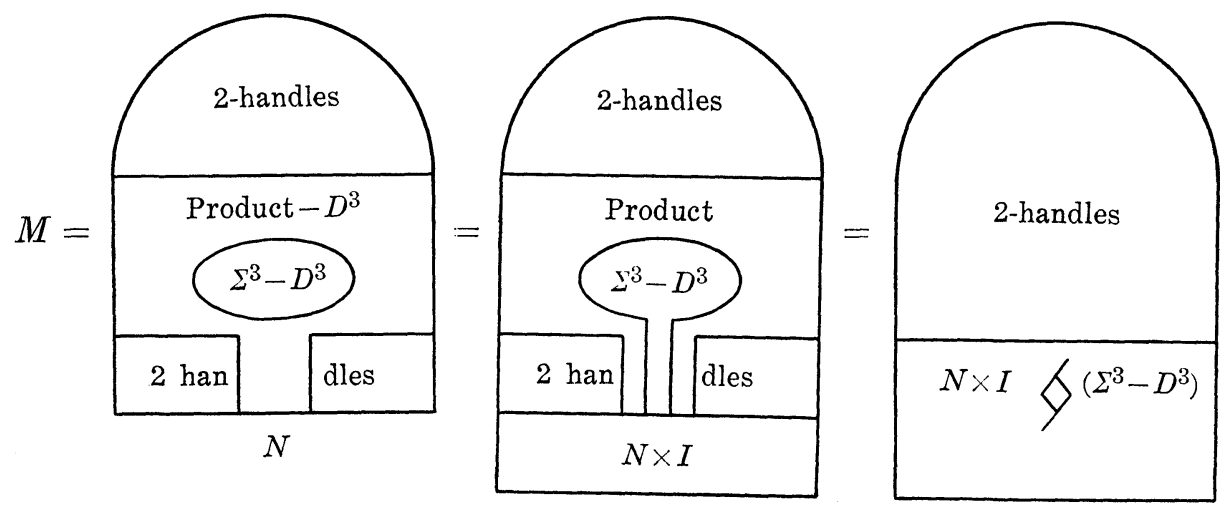

Set $\bar{N}=N \times I \models\left(\Sigma^{3}-D^{3}\right)$.

This completes the proof of Theorem 1 . 
Note. The orientation restriction in dimension 3 results from ignorance about $h$-cobordisms on $R P^{2}$.

Corollary. Let $M$ be a compact simply connected P. L.

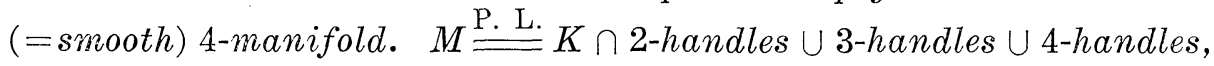
where $K$ is a compact contractable 4-manifold.

Proof. Apply Theorem 1 to $\left(M-D^{4}, \partial D^{4}\right)$. Let $K=\bar{N} \cup D^{4}$.

REMARK. A. Casson has recently exhibited (unpublished work) a simply connected P. L. 4-manifold with boundary, $M$, with the property that every handle decomposition of $M, \mathscr{C}(M)$, must contain a 1 handle. This answers negatively a question raised in [4] on the existence of (relative) 2 -spines. So the preceding corollary is all one can hope for.

Proof of Theorem 2. Let $M \stackrel{\text { P. L. }}{=} K \cup 2$-handles $\cup 3$-handles $\cup 4$ handles. Let $\hat{M}=$ cone $(\partial K) \cup 2$-handles $\cup$ 3-handles $\cup 4$-handles. $H_{2}(M ; Z) \cong H_{2}(\hat{M}$, cone $(\partial K) ; Z) \cong H_{2}(\hat{M} ; Z)$. Any element of $H_{2}(\hat{M}$, cone $(\partial K) ; Z)$ is represented by a relatively imbedded 2-disk constructed as a linear combination of 2 -handles in the above handle decomposition by taking ambient boundary-connected-sums. So every element, $\alpha$, of $H_{2}(\hat{M} ; Z)$ is represented by a simplicial imbedding, $\omega$, of $S^{2}$ in $\hat{M}$. By a theorem of R. Edwards, [1], (cone $\left.\partial K\right) \times R$ is (topologically) homeomorphic to $K \times R, \hat{M} \times R$ is (topologically) homeomorphic to $M \times R$. The composition:

$$
S^{2} \times R \stackrel{\omega \times \mathrm{id}_{R}}{\longrightarrow} \hat{M} \times R \stackrel{\text { top. homeomorphism }}{\longrightarrow} M \times R
$$

is the topological imbedding with the claimed properties.

\section{REFERENCES}

1. R. Edwards, Unpublished results on the double suspension conjecture.

2. M. Fredman, A converse to (Milnor-Kervaire theorem) $\times R$, etc. $\cdots$, to appear.

3. J. Milnor, Lectures on the h-cobordism theorem, Princeton Math. Series, (1965).

4. J. Milnor and M. Kernaire, On 2-spheres in 4-manifolds, Proc. Nat. Acad. Sci. U.S., 47 (1961), 1651-1657.

5. J. Stallings, On Fibering Certain 3-Manifolds, Topology of 3-Manifolds and Related Topics, Prentice-Hall, Inc., Englewood Cliffs, N.J., 1962, 95-100.

Received October 20, 1976 and in revised form August 9, 1977. Author partially supported by NSF Grant MPS 72-05055 A03.

University of California, San Diego

LA JOLLA, CA 92037 


\section{PACIFIC JOURNAL OF MATHEMATICS}

\section{EDITORS}

RICHARD ARENS (Managing Editor)

University of California

Los Angeles, California 90024

C. W. CURTIS

University of Oregon

Eugene, OR 97403

C. C. MOORE

University of California

Berkeley, CA 94720
J. DugundjI

Department of Mathematics University of Southern Californı Los Angeles, California 90007

R. Finn and J. Milgram Stanford University Stanford, California 94305

ASSOCIATE EDITORS

E. F. BECKENBACH

B. H. NeUmanN

F. WOLF

K. YoSHIDA

\section{SUPPORTING INSTITUTIONS}

UNIVERSITY OF BRITISH COLUMBIA CALIFORNIA INSTITUTE OF TECHNOLOGY

UNIVERSITY OF CALIFORNIA

MONTANA STATE UNIVERSITY

UNIVERSITY OF NEVADA, RENO

NEW MEXICO STATE UNIVERSITY

OREGON STATE UNIVERSITY

UNIVERSITY OF OREGON
UNIVERSITY OF SOUTHERN CALIFORNIA

STANFORD UNIVERSITY

UNIVERSITY OF HAWAII

UNIVERSITY OF TOKYO

UNIVERSITY OF UTAH

WASHINGTON STATE UNIVERSITY

UNIVERSITY OF WASHINGTON 


\section{Pacific Journal of Mathematics}

\section{Vol. 80, No. $1 \quad$ September, 1979}

Jeroen Bruijning and Jun-iti Nagata, A characterization of covering dimension by

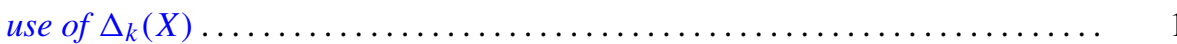

John J. Buoni and Albert Jonathan Klein, On the generalized Calkin algebra ...... 9

Thomas Ashland Chapman, Homotopy conditions which detect simple homotopy

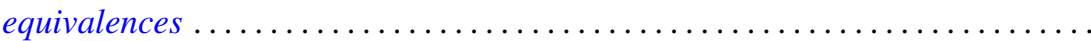

John Albert Chatfield, Solution for an integral equation with continuous interval

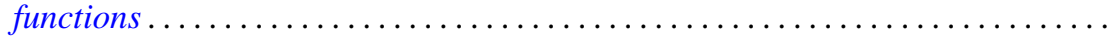

Ajit Kaur Chilana and Ajay Kumar, Spectral synthesis in Segal algebras on

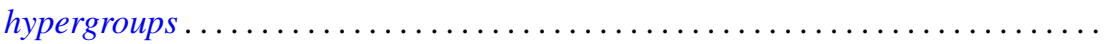

Lung O. Chung, Jiang Luh and Anthony N. Richoux, Derivations and

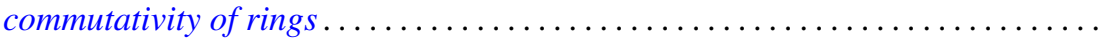

Michael George Cowling and Paul Rodway, Restrictions of certain function spaces to closed subgroups of locally compact groups .....................

David Dixon, The fundamental divisor of normal double points of surfaces........

Hans Georg Feichtinger, Colin C. Graham and Eric Howard Lakien,

Nonfactorization in commutative, weakly selfadjoint Banach algebras . . . . . . .

Michael Freedman, Cancelling 1-handles and some topological imbeddings ....... .

Frank E., III Gerth, The Iwasawa invariant $\mu$ for quadratic fields . . . . . . . . . . . . . .

Maurice Gilmore, Three-dimensional open books constructed from the identity

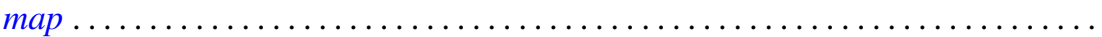

Stanley P. Gudder, A Radon-Nikodým theorem for $*$-algebras .

Peter Wamer Harley, III and George Frank McNulty, When is a point Borel? .

Charles Henry Heiberg, Fourier series with bounded convolution powers . .

Rebecca A. Herb, Characters of averaged discrete series on semisimple real Lie

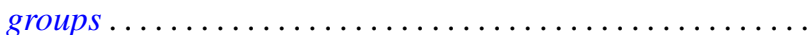

Hideo Imai, On singular indices of rotation free densities . .

Sushil Jajodia, On 2-dimensional CW-complexes with a single 2-cell . . .

Herbert Meyer Kamowitz, Compact operators of the form $u C_{\varphi}$

Matthew Liu and Billy E. Rhoades, Some properties of the Chebyshev method...

213

George Edgar Parker, Semigroups of continuous transformations and generating

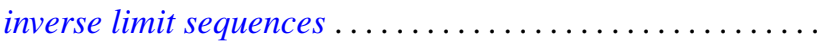

Samuel Murray Rankin, III, Oscillation results for a nonhomogeneous

equation ...

Martin Scharlemann, Transverse Whitehead triangulations ...

Gary Joseph Sherman, A lower bound for the number of conjugacy classes in a

finite nilpotent group

Richard Arthur Shoop, The Lebesgue constants for $\left(f, d_{n}\right)$-summability .

Stuart Jay Sidney, Functions which operate on the real part of a uniform

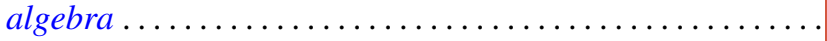

Tim Eden Traynor, The group-valued Lebesgue decomposition 